\title{
Median-Prefiltering-Based Robust Acquisition of Direct-Sequence Spread-Spectrum Signals in Wide-Band Pulse Jamming
}

\author{
Yong-Hwan Lee, Member, IEEE, and Seung-Jun Kim, Student Member, IEEE
}

\begin{abstract}
We propose robust acquisition schemes for chip time-synchronous direct-sequence spread-spectrum (DS/SS) signals in wide-band pulse jamming. To mitigate the performance degradation due to the impulse-like wide-band jamming signal, the received signal is preprocessed by employing simple order statistic filters. We consider the use of two types of median filtering schemes: a running median filter operating at a chip rate and a block median filter processing the received signal sampled at an oversampling rate. For analytic design, the partial correlation of median filtered pseudonoise (PN) signals is approximated by a piecewise linear model using an upper bound. The analytic design is verified by computer simulation. Finally, the acquisition performance of the proposed schemes is compared to that of conventional schemes.
\end{abstract}

Index Terms-Median filter, robust acquisition, spread spectrum, wide-band jamming.

\section{INTRODUCTION}

D IRECT-sequence spread-spectrum (DS/SS) communication systems are known to have inherent immunity to tone jamming or narrow-band jamming [1]. When the jamming signal power is high, however, the performance of a DS/SS system can be severely degraded by wide-band pulse jamming [2]. In particular, a wide-band jamming signal with an optimized duty cycle can significantly increase the bit error rate (BER) of the system. When the performance is compared in terms of the BER, it is shown that the effect of the impulse noise is equivalent to that of four additional users in an additive white Gaussian noise (AWGN) channel with the same signal-to-noise power ratio (SNR) [3]. Gui and $\mathrm{Ng}$ [4] analyzed performance degradation in a DS/SS system due to wide-band jamming. When the processing gain is 255 and the power ratio of the jamming signal to the background Gaussian noise is 100, they showed that performance degradation can be up to about $15 \mathrm{~dB}$ at a BER of $10^{-6}$.

The performance may be improved by employing various kinds of linear and/or nonlinear processing techniques [5]. It has been recognized that nonlinear processing techniques are more effective than linear ones for handling non-Gaussian noise. So

Manuscript received October 6, 1998; revised August 8, 2001. This work was supported in part by the Korea Science and Engineering Foundation.

Y.-H. Lee is with the School of Electrical Engineering, Seoul National University, 151-742 Seoul, Korea.

S. J. Kim is with the University of California at Santa Barbara, Santa Barbara, CA 93106 USA.

Publisher Item Identifier S 0018-9545(02)00439-5. far, most of the research has concentrated on the use of memoryless nonlinear processing [6]. Aazhang and Poor showed that the use of a hard limiter as a preprocessor can improve the performance of a DS/SS receiver as much as $9 \mathrm{~dB}$ in a single-user environment [7]. However, they also reported that the linear correlator performs better than the hard limiter as the multiple access noise increases. Stüber [8] reported that the performance of a coded DS/DPSK receiver employing a soft limiter in the worst pulse jamming environment is only about $0.5 \mathrm{~dB}$ inferior to that of the receiver employing the maximum likelihood soft-decision decoder with the use of perfect jamming information. Chao and Lee [9] proposed an optimum memoryless nonlinear device that outperforms both the hard limiter and the soft limiter in wide-band jamming environment by applying Dempster-Shafer theory. However, the implementation may become a little complicated due to the use of an exponential function.

Although there has been some research on the acquisition of pseudonoise (PN) signals in DS/SS systems [10]-[12], few works have addressed the acquisition problem in additive non-Gaussian noise environment. Siess and Weber [13] analyzed acquisition performance under tone jamming and designed a receiver with constant false-alarm rate by estimating the jammer-to-signal power ratio. Since they assumed an ideal estimator, however, the receiver performance may be affected depending upon the accuracy of the estimator.

It is well known that the use of linear correlators yields the optimum acquisition performance in the AWGN channel. However, the linear scheme has rapid degradation in acquisition performance when a non-Gaussian wide-band jamming signal exists. The use of nonlinear processing methods may provide acquisition performance robust to the existence of non-Gaussian noise. In addition, the use of nonparametric preprocessors is preferred since the characteristics of such jamming signals are not exactly known.

The limiter is a well-known nonlinear memoryless processor whose transfer function is given by

$$
g(x)= \begin{cases}x, & |x|<\eta \\ \eta, & x>\eta \\ -\eta, & x<-\eta .\end{cases}
$$

When this soft limiter is applied to a non-Gaussian noise environment, the optimum threshold $\eta$ is dependent upon the characteristics of the jamming signal. For example, unless the power of the jamming signal is known a priori, it may not be feasible 


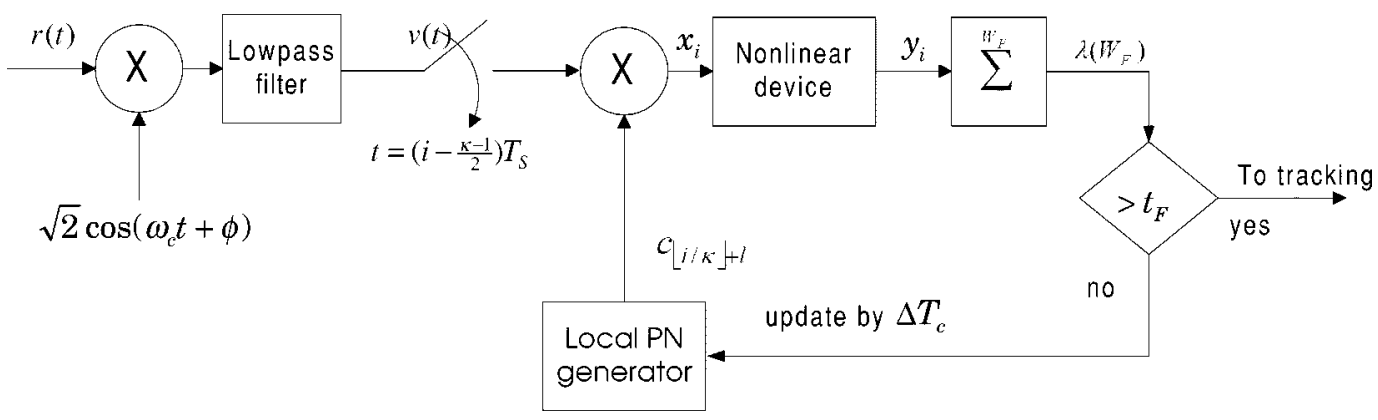

Fig. 1. Acquisition scheme employing a nonlinear preprocessor.

to design the optimum value of $\eta$. As the limiting case of a soft limiter, the transfer function of a hard limiter is defined by

$$
g(x)= \begin{cases}-1, & x<0 \\ 1, & x \geq 0\end{cases}
$$

The sign detector, which employs a hard limiter as a preprocessor, is widely used because it requires minimum knowledge of the signal that the noise has a zero mean and symmetric probability density function (pdf). The sign detector is simple for implementation, but its performance is too inferior to that of the linear detector in the absence of a jamming signal.

The use of nonlinear processors with memory can further improve the performance under non-Gaussian noise. It has been known that Wilcoxon detector is robust to impulse noise while providing good performance in the AWGN. However, the implementation complexity rapidly increases as the sample size increases since it needs to sort the absolute magnitudes of the samples [14].

Recently, order statistic filters (OSFs) have been successfully applied to robust signal-processing and detection areas [15], [16]. In particular, the median filter, a simple OSF, has been shown to be effective against impulse noise while keeping the implementation complexity relatively low. It was successfully applied to the design of robust detectors in a non-Gaussian noise environment [17], [18]. Iinatti [19] considered the use of a matched filter for acquisition of DS/SS signals in pulsed tone jamming with the aid of a median filter. But the analytical design is difficult, and the use of the matched filter somewhat complicates the implementation.

In this paper, we consider the use of median filters as a preprocessor to design a robust acquisition scheme for DS/SS signals. In Section II, we describe the model of the DS/SS system for PN signal acquisition. Section III discusses the charateristics of the median filtered PN signals. Based on an approximate model of the partial correlation of the PN sequences, we analytically design the detector for phase alignment in Section IV. Lastly, the analytic design is verified by computer simulation in Section V.

\section{SySTEM MODELING FOR ACQUISITION}

Assume that the data signal is represented by

$$
d(t)=\sum_{i=-\infty}^{\infty} b_{i} P_{T}(t-i T)
$$

where $b_{i} \in\{-1,1\}$ represents the $i$ th binary data, $P_{T}(\cdot)$ is the shaping pulse given by

$$
P_{T}(t)= \begin{cases}1, & -\frac{T}{2} \leq t<\frac{T}{2} \\ 0, & \text { otherwise }\end{cases}
$$

and $T$ is the bit duration interval. The PN spreading waveform $c(t)$ can be represented by

$$
c(t)=\sum_{j=-\infty}^{\infty} c_{j} P_{T_{c}}\left(t-j T_{c}\right)
$$

where $\left\{c_{j}\right\}$ is the spreading sequence with period $N$ and $T_{c}$ is the chip duration time. Assuming no modulation during the acquisition period, say, $b_{i}=1$, the transmitted signal $s(t)$ can be expressed by, for some $k$

$$
s(t)=\sqrt{2 P} c\left(t+k T_{c}\right) \cos \left(\omega_{c} t+\varphi\right)
$$

where $P$ is the transmitted power and $\omega_{c}$ and $\varphi$ are the angular frequency and the phase of the carrier, respectively.

The received signal corrupted by wide-band jamming signal can be represented by

$$
r(t)=\sqrt{2 P} c\left(t+k T_{c}-\tau\right) \cos \left(\omega_{c} t+\phi\right)+\hat{G}(t)+\hat{J}(t)
$$

where $\hat{G}(t)$ is AWGN with two-sided power spectral density $N_{0} / 2, \hat{J}(t)$ is the wide-band jamming interference signal with two-sided power spectral density $N_{J} / 2, \tau$ is the propagation delay caused by the channel, and $\phi=\varphi-\omega_{c} \tau$. For simplicity of description, we assume that the chip timing is known so that we can let $\tau=0$ without loss of generality [20]. If the chip timing is not correctly obtained, the chip SNR may substantially deteriorate.

We consider the use of a coherent demodulation scheme for ease of analytic design. Note that the proposed idea can also be applied to noncoherent schemes with little modification. The output of the ideal low-pass filter with cutoff frequency $f_{b}(=$ $1 / T_{c}$ ) after coherent demodulation can be represented as

$$
v(t)=\sqrt{P} c\left(t+k T_{c}\right)+G(t)+J(t)
$$

where $G(t)$ and $J(t)$ represent the low-pass filtered noise and jamming signal terms. As shown in Fig. 1, the demodulated signal $v(t)$ is oversampled at a sampling rate of $f_{s}\left(=\left(1 / T_{s}\right)\right)=$ 
$\kappa f_{b}, \kappa \geq 1$, and then multiplied by the local PN signal for correlation. Let $x_{i}(i \geq 0)$ be the output of the multiplier

$$
\begin{aligned}
x_{i} & =\left.v(t)\right|_{t=\left(i-\frac{\kappa-1}{2}\right) T_{s}} \cdot c_{\lfloor i / \kappa\rfloor+l} \\
& =s_{i}+z_{i}
\end{aligned}
$$

where $\lfloor t\rfloor$ represents the maximum integer that is not larger than $t, l T_{c}$ represents the phase of the local PN signal, and $s_{i}$ and $z_{i}$ denote the signal and the noise terms, respectively

$$
\begin{aligned}
& s_{i}=\sqrt{P} c_{\lfloor i / \kappa\rfloor+k} c_{\lfloor i / \kappa\rfloor+l} \\
& z_{i}=n_{i} c_{\lfloor i / \kappa\rfloor+l} .
\end{aligned}
$$

Here

$$
\left.n_{i}=G\left[(i-(\kappa-1) / 2) T_{s}\right)\right]+J\left[(i-(\kappa-1) / 2) T_{s}\right] .
$$

We assume that the signal power is normalized, say, $P=1$.

Assuming that the interference noise occurs at a duty factor of $\epsilon$, the additive noise terms can be modeled using Huber's $\epsilon$-contaminated model [21]. The pdf of the equivalent noise term $z_{i}$ can be represented by

$$
f_{z}(z)=(1-\epsilon) f_{G}(z)+\epsilon f_{J}(z), \quad 0 \leq \epsilon<1
$$

where $f_{G}(z)$ is the pdf of the background Gaussian noise with variance $\sigma_{G}^{2}=N_{0} f_{b}$ and $f_{J}(z)$ is the pdf of the jamming noise with the total variance $\sigma_{J}^{2}=N_{J} f_{b} \gg \sigma_{G}^{2}$. Then, the variance of the random variable $z_{i}$ is given by $\sigma_{z}^{2}=(1-\epsilon) \sigma_{G}^{2}+\epsilon \sigma_{J}^{2}$. We define the signal-to-jammer power ratio (SJR) by $\left(P / \sigma_{J}^{2}\right)$. This model, similar to that used in [8], represents the case where the receiver is affected by a jamming signal with probability $\epsilon$. Note that this jamming noise model is different from the on-off pulse jamming noise model with a duty factor of $\epsilon$ in [4]. Since the occurrence of jamming noise is not predictable, the estimation method proposed in [22] may not be applicable.

When the samples are taken at a chip rate, i.e., $f_{s}=f_{b}, s_{i}$ and $z_{i}$ are expressed as

$$
\begin{aligned}
s_{i} & =\sqrt{P} c_{i+k} c_{i+l} \\
z_{i} & =\left\{\tilde{G}\left(i T_{s}\right)+\tilde{J}\left(i T_{s}\right)\right\} c_{i+l} .
\end{aligned}
$$

It can be easily shown that $\left\{z_{i}\right\}$ are uncorrelated random variables provided that $\left\{c_{i}\right\}$ are independent and identically distributed (i.i.d.) and the low-pass filter is ideal. Since the probability distribution is not Gaussian, the uncorrelatedness does not imply the independence. However, we assume that $z_{i}$ are mutually independent for ease of analytic design. The multiplier outputs $x_{i}$ are preprocessed by using a preprocessor

$$
y_{i}=g\left(x_{i}\right) \text {. }
$$

It has been shown that the acquisition problem can be formulated as a detection problem testing a simple hypothesis against a simple alternative [2]. Let the hypothesis $H_{1}$ be the case when the phase of the two PN sequences is aligned, i.e., when $k=l$, and the hypothesis $H_{0}$ when $k \neq l$. The hypothesis decision is made by comparing the test statistic

$$
\lambda(w)=\sum_{i=0}^{w-1} h\left(y_{i}\right)
$$

with a threshold $t_{F}$, where $h(\cdot)$ is some linear function. In this paper, we consider a fixed sample size test in which the number $w$ of test samples is set to a fixed number $W_{F}$.

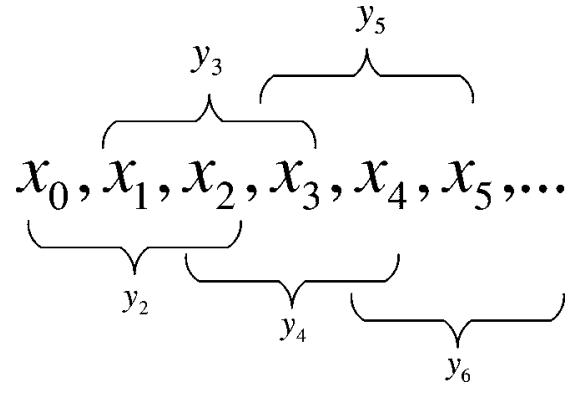

(a)

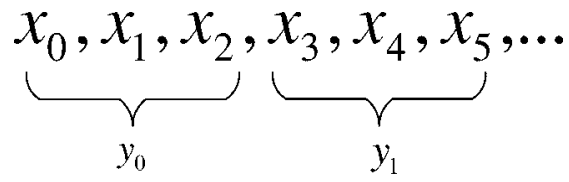

(b)

Fig. 2. Two median filtering processes. (a) Running median filtering. (b) Block median filtering

When the hypothesis $H_{0}$ is declared, the phase of the local PN sequence is updated by $\Delta T_{c}$. This process is repeated until the acquisition is achieved. The constant $\Delta$ usually has some fractional value like 1 or $1 / 2$, depending on the pull-in range of the tracking circuit and/or whether the chip timing is available to the receiver. Since we have assumed that the chip timing is known to the receiver, it suffices to use $\Delta=1$.

\section{Modeling of Median Filtered PN Signals}

We consider two types of median filtering processors. The running median filter can be defined by [23]

$$
\begin{aligned}
y_{i} & =g_{R}\left(x_{i}\right) \\
& =\operatorname{MED}_{M_{R}}\left(x_{i}, x_{i-1}, \ldots, x_{i-M_{R}+1}\right), \quad i \geq M_{R}-1
\end{aligned}
$$

where $\operatorname{MED}_{M_{R}}(\cdot)$ denotes a median filter with window size $M_{R}$. We define the block median filter by

$$
\begin{aligned}
y_{i} & =g_{B}\left(x_{i}\right) \\
& =\operatorname{MED}_{M_{B}}\left(x_{i M_{B}}, x_{i M_{B}+1}, \ldots, x_{i M_{B}+M_{B}-1}\right) .
\end{aligned}
$$

Fig. 2 illustrates the outputs of the two median filters when $M_{R}$ and $M_{B}$ are all equal to three.

The oversampled multiplier outputs corresponding to the $i$ th chip are first processed by a block median filter $g_{B}(\cdot)$. For ease of implementation, we consider the use of a window size $M_{B}$ equal to the oversampling ratio $\kappa$. Then, the output of $g_{B}$ is processed by a running median filter at a chip rate.

First we consider the case when the samples are taken at a chip rate $(\kappa=1)$. That is, the samples are processed only by a running median filter without the use of block median filtering. Define $T(w)$ by a partial sum of the running median filter outputs

$$
\begin{aligned}
T(w) & =\sum_{i=0}^{w-1} g_{R}\left(x_{i+M_{R}-1}\right) \\
& =\sum_{i=0}^{w-1} g_{R}\left(s_{i+M_{R}-1}+z_{i+M_{R}-1}\right) .
\end{aligned}
$$




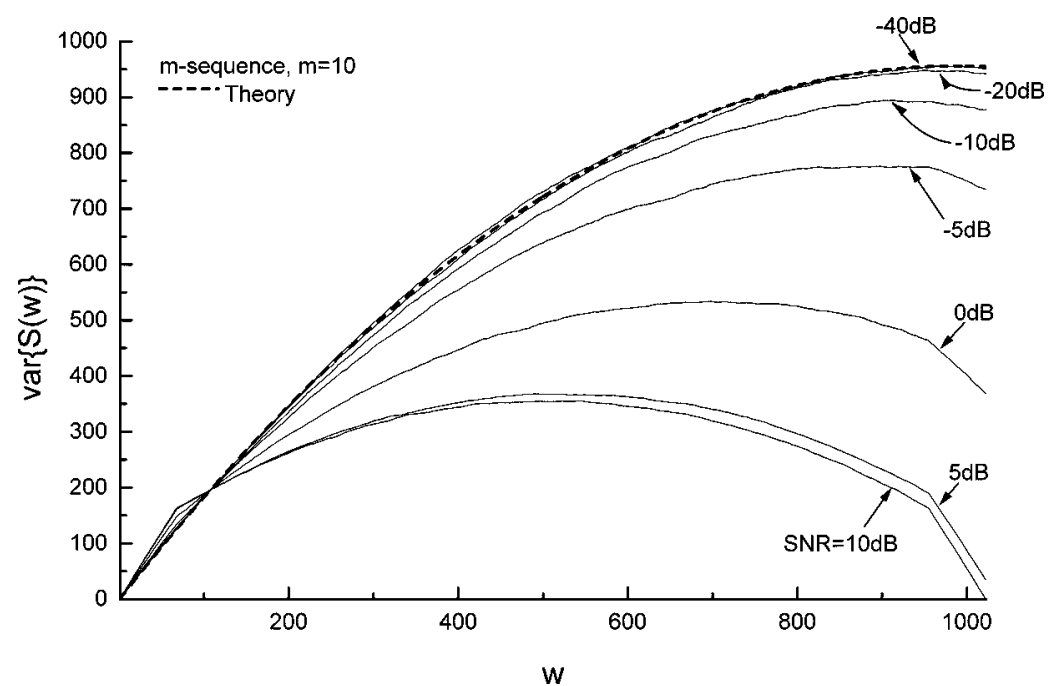

Fig. 3. Variance of $S(w)$ when Gaussian noise is added.

Due to the nonlinear property of the median filter, the median filtered output of the sum of the signal component $s_{i}$ and the noise component $z_{i}$ is not the same as the sum of the median filtered signal term and the median filtered noise term. However, the median filter output $y_{i}$ can be expressed as

$$
\begin{aligned}
y_{i} & =g_{R}\left(s_{i+M_{R}-1}+z_{i+M_{R}-1}\right) \\
& =s_{i+M_{R}-1}^{\prime}+z_{i+M_{R}-1}^{\prime}
\end{aligned}
$$

where

$$
\begin{aligned}
& s_{i+M_{R}-1}^{\prime} \in\left\{s_{i+M_{R}-1}, \ldots, s_{i+1}, s_{i}\right\} \\
& z_{i+M_{R}-1}^{\prime} \in\left\{z_{i+M_{R}-1}, \ldots, z_{i+1}, z_{i}\right\} .
\end{aligned}
$$

Therefore, $T(w)$ can be written as

$$
\begin{aligned}
T(w) & =\sum_{i=0}^{w-1} s_{i+M_{R}-1}^{\prime}+\sum_{i=0}^{w-1} z_{i+M_{R}-1}^{\prime} \\
& =S(w)+Z(w)
\end{aligned}
$$

where

$$
\begin{aligned}
& S(w)=\sum_{i=0}^{w-1} s_{i+M_{R}-1}^{\prime} \\
& Z(w)=\sum_{i=0}^{w-1} z_{i+M_{R}-1}^{\prime}
\end{aligned}
$$

The signal component $S(w)$ is equal to $w$ under hypothesis $H_{1}$. Under hypothesis $H_{0}, S(w)$ can be approximated as a Gaussian random variable by invoking the central limit theorem. Thus, it suffices to calculate the mean and the variance of $S(w)$.

The pattern of $-1 \mathrm{~s}$ and $1 \mathrm{~s}$ in the output sequence is dependent upon the pattern of the input sequence due to the use of memory by the median filter. However, when the noise power is much larger than the signal power (i.e., $\sigma_{z}^{2} \gg P$ ), the pattern of the output sequence becomes quite independent of the original pattern because the statistically independent noise term $z_{i}$ dominates the signal term $x_{i}$. In this case, it can be shown that the mean value of $S(w)$ is

$$
E\{S(w)\}=-\frac{w}{N}
$$

Taking into consideration the correlation between the output samples $s_{i}^{\prime}$ and $s_{j}^{\prime}$ for $|i-j|<M_{R}$, we can have

$$
\begin{aligned}
E\left\{S^{2}(w)\right\}= & \sum_{i=M_{R}-1}^{w+M_{R}-2} \sum_{j=M_{R}-1}^{w+M_{R}-2} E\left\{s_{i}^{\prime} s_{j}^{\prime}\right\} \\
= & w E\left\{s_{i}^{\prime 2}\right\}+2 \sum_{p=1}^{M_{R}-1}(w-p) E\left\{s_{i}^{\prime} s_{i+p}^{\prime}\right\} \\
& +\left(w-M_{R}\right)\left(w-M_{R}+1\right) \\
& \times E\left\{s_{i}^{\prime} s_{j}^{\prime},|i-j| \geq M_{R}\right\} \\
= & w+2 \sum_{p=1}^{M_{R}-1}(w-p) E\left\{s_{i}^{\prime} s_{i+p}^{\prime}\right\} \\
& +\left(w-M_{R}\right)\left(w-M_{R}+1\right)\left(-\frac{1}{N}\right)
\end{aligned}
$$

where the values of $E\left\{s_{i}^{\prime} s_{i+p}^{\prime}\right\}$ for $1 \leq p<M_{R}$ can be calculated by applying the method described in [24]. For example, when $M_{R}=3$, suppose that the inputs to the median filter are $x_{i-2}, x_{i-1}, x_{i}$, and $x_{i+1}$ and the corresponding outputs are $y_{i}$ and $y_{i+1}$. Since it can be shown that there are eight cases when $y_{i}=y_{i+1}$ and 16 cases when $y_{i} \neq y_{i+1}$

$$
E\left\{s_{i}^{\prime} s_{i+1}^{\prime}\right\}=\frac{8}{24}+\frac{16}{24}\left(-\frac{1}{N}\right)=\frac{1}{3}-\frac{2}{3 N} .
$$

Similarly, when the input samples are $x_{i-2}, x_{i-1}, x_{i}, x_{i+1}$, and $x_{i+2}$, there are only 16 cases where $y_{i}=y_{i+2}$ among a total of 120 possible cases. Thus, we have

$$
E\left\{s_{i}^{\prime} s_{i+2}^{\prime}\right\}=\frac{16}{120}+\frac{104}{120}\left(-\frac{1}{N}\right)=\frac{2}{15}-\frac{13}{15 N} .
$$

When $M_{R}=3$, the variance of $S(w)$ is given by

$$
\begin{aligned}
\operatorname{var}\{S(w)\}= & w+2(w-1)\left(\frac{1}{3}-\frac{2}{3 N}\right) \\
& +2(w-2)\left(\frac{2}{15}-\frac{13}{15 N}\right) \\
& +(w-3)(w-2)\left(-\frac{1}{N}\right)-\left(\frac{w}{N}\right)^{2} .
\end{aligned}
$$

Fig. 3 depicts the actual values of $\operatorname{var}\{S(w)\}$ when $M_{R}=3$ under additive Gaussian noise condition, where the analytical 


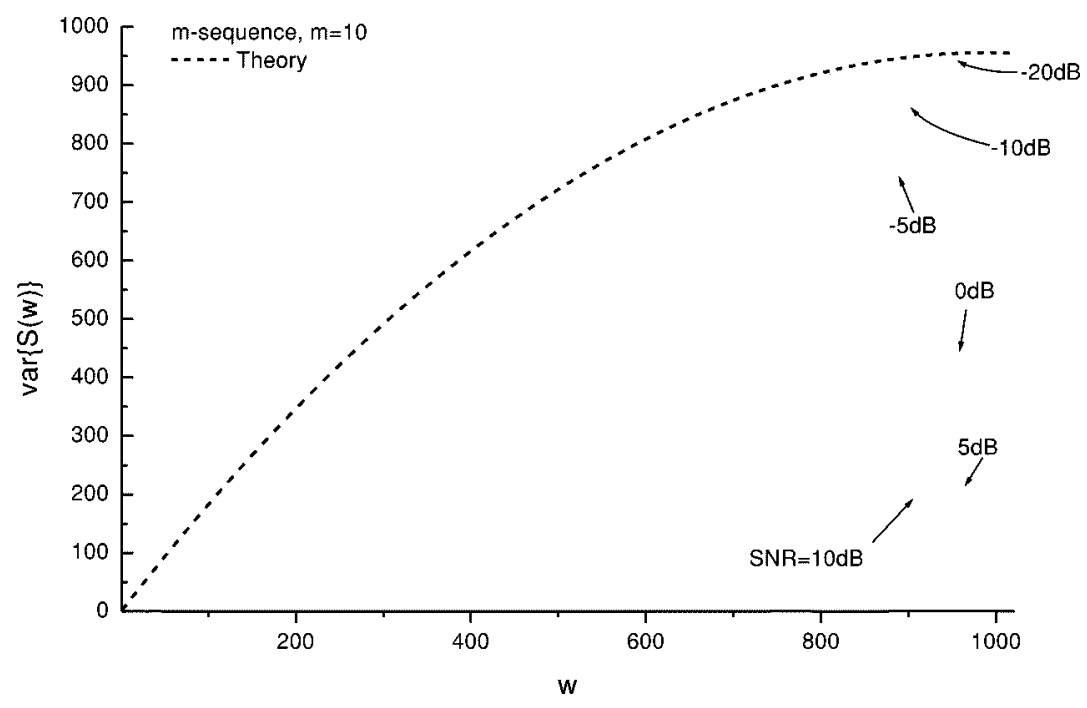

Fig. 4. Variance of $S(w)$ when Laplacian noise is added.

TABLE I

GENERATING POLYNOMials

\begin{tabular}{c|c}
\hline Sequence & Generating polynomial \\
\hline $\mathrm{A}$ & $1+x^{4}+x^{9}$ \\
\hline $\mathrm{B}$ & $1+x^{3}+x^{10}$ \\
\hline
\end{tabular}

result at low SNR is depicted as a dotted line for comparison, $m$ denotes the number of shift registers of the $m$-sequence generator, and the period of the corresponding sequence is $2^{m}-1$.The $m$-sequences are obtained by generating polynomials tabulated in Table I. Fig. 4 plots $\operatorname{var}\{S(w)\}$ when $M_{R}=3, m=10$ and the Laplacian noise is added. It can be seen that the theoretical variance agrees well with the actual variances as the SNR decreases. Since the SNR per chip is usually very low in a DS/SS system, the use of approximated analytical results can be applicable to analytic design of an acquisition system.

To build a conservative model for $S(w)$ under hypothesis $H_{0}$, we consider the use of an approximate upper bound $\hat{S}(w)$ based on $95 \%$ confidence interval defined as

$$
\hat{S}(w)=E\{S(w)\}+1.65 \sqrt{\operatorname{var}\{S(w)\}} .
$$

Fig. 5 depicts the actual maximum values and the proper upper bound obtained by experiments. The experimental values are obtained after median filtering the $m$-sequences generated by the polynomial A in Table I under additive Gaussian noise condition at $-20 \mathrm{~dB}$ SNR. It can be seen that the analytical results agree well with the experimental results. However, the curved shape of $\hat{S}(w)$ implies that the signal model under hypothesis $H_{0}$ is not i.i.d., which makes the analysis and design of the detector quite complicated.

To alleviate this problem, we consider the use of a simple two-piece linearized model given as

$$
\bar{S}(w)= \begin{cases}0.75 w, & w \leq W_{b} \\ \max _{w} \hat{S}(w), & w>W_{b}\end{cases}
$$

where $W_{b}$ is the breakpoint of the two segments. Here, the slope of $\bar{S}(w)$ for $w \leq W_{b}$ is set to a constant 0.75 by considering the largest correlation coefficient under hypothesis $H_{0}$ when $\Delta=$

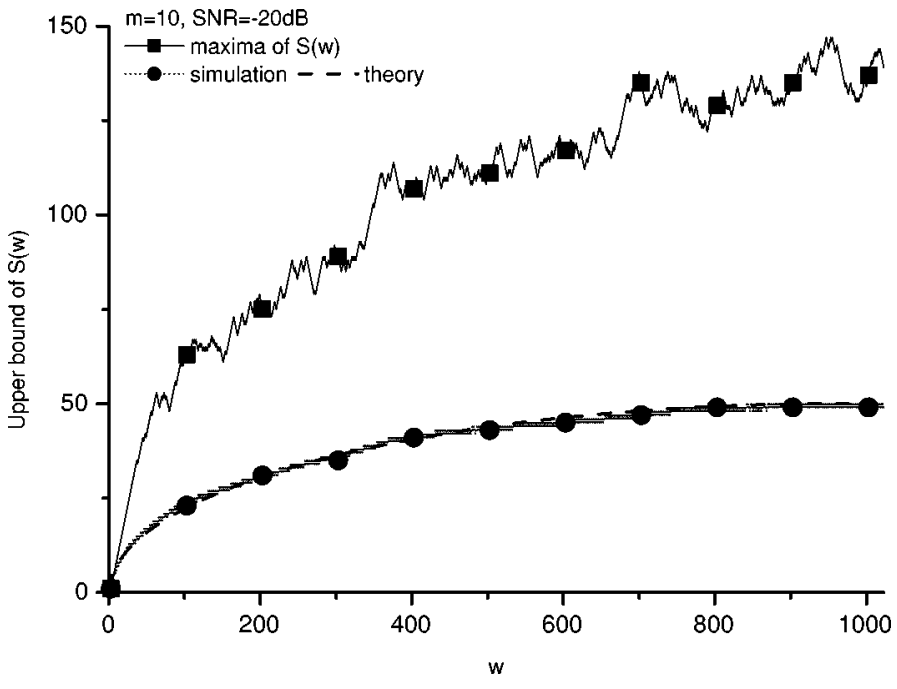

Fig. 5. An upper bound of $S(w)$.

0.5 . Since $\hat{S}(w)$ has a maximum value approximately when $w \approx$ $N$, the value of $W_{b}$ is determined by

$$
0.75 W_{b}=\hat{S}(N) \text {. }
$$

As an example, Fig. 6 depicts $\bar{S}(w)$ and $\hat{S}(w)$ when $m=9$. It can be seen that $\bar{S}(w)$ provides a valid upper bound, except for small values of $w$. Using the linearized approximate model for $s^{\prime}$ under hypothesis $H_{0}$, we can approximate the mean value of $s_{i}$ by $\hat{s}^{\prime}$. Since $s_{i+M_{R}-1}^{\prime}=1$ under hypothesis $H_{1}, \hat{s}^{\prime}$ can be approximated as

$$
\hat{s}^{\prime}= \begin{cases}\hat{s}_{1}=1, & \text { when } k=l \\ \hat{s}_{0 i}= \begin{cases}\hat{s}_{a}=0.75, & i \leq W_{b} \\ \hat{s}_{b}=0.0, & i>W_{b},\end{cases} & \text { when } k \neq l .\end{cases}
$$

The noise component $Z(w)$ should also be properly modeled to analytically design the detector. When the phases of two PN sequences are aligned, i.e., $s_{i}=s_{i+1}=\cdots=s_{i+M_{R}-1}=1$

$$
z_{i}^{\prime}=g_{R}\left(z_{i}\right)
$$

Then, the pdf of $z_{i}^{\prime}$ is given by [25]

$$
f_{z^{\prime}}\left(z^{\prime}\right)=3 ! F_{z}\left(z^{\prime}\right)\left[1-F_{z}\left(z^{\prime}\right)\right] f_{z}\left(z^{\prime}\right)
$$




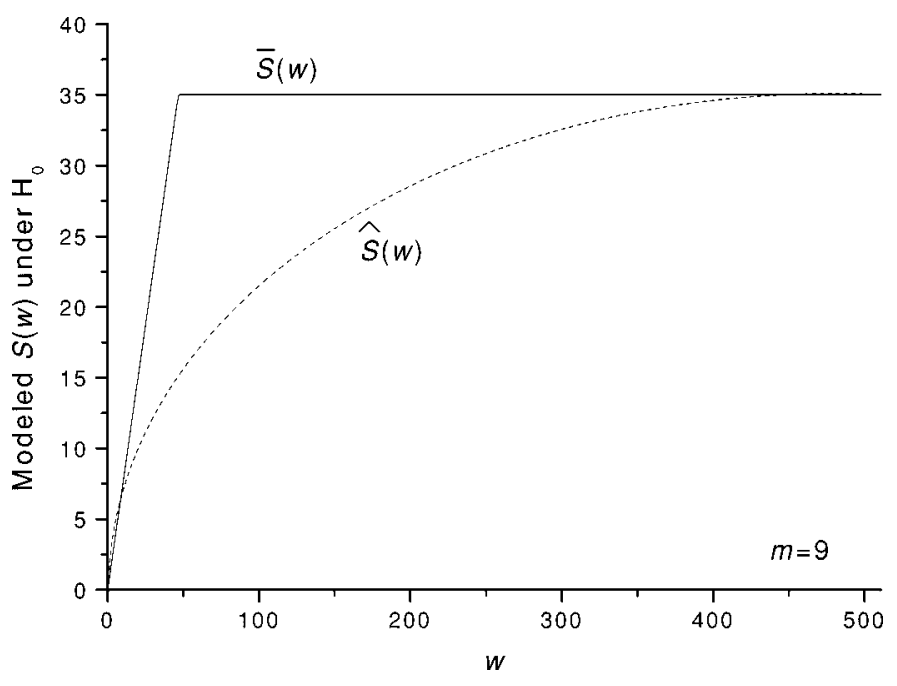

Fig. 6. $\quad \bar{S}(w)$ and $\hat{S}(w)$ when $m=9$.

where $F_{z}\left(z^{\prime}\right)$ denotes the cumulative distribution function of $z_{i}$. It can be shown that $Z(w)$ has zero mean and the variance given by

$$
\operatorname{var}\{Z(w)\}=\sigma_{z^{\prime}}^{2}\left[w+2 \sum_{\ell=1}^{M_{R}-1}(w-\ell) \rho_{z}(\ell)\right]
$$

where $\rho_{z}(\ell)$ is given by

$$
\rho_{z}(\ell)=\frac{E\left\{z_{i} z_{i+\ell}\right\}}{\sigma_{z^{\prime}}^{2}}
$$

and $\sigma_{z^{\prime}}^{2}$ is the power of $z_{i}^{\prime}$, calculated by

$$
\sigma_{z^{\prime}}^{2}=\int_{-\infty}^{\infty} z^{2} f_{z^{\prime}}(z) d z
$$

It can be approximated by [18]

$$
\sigma_{1}^{2} \triangleq \lim _{w \rightarrow \infty} \frac{\operatorname{var}\{Z(w)\}}{w}=\sigma_{z^{\prime}}^{2}\left[1+2 \sum_{\ell=1}^{M_{R}-1} \rho_{z}(\ell)\right] .
$$

This appoximation implies that the variance of the noise component is assumed to be a little larger than the true one because $\operatorname{var}\{Z(w)\}$ is a monotonically increasing function of $w$.

When $k \neq l$, i.e., under hypothesis $H_{0}$, the variance of the noise component is somewhat larger than $\sigma_{1}^{2}$ because the partial sum of the signal component has magnitude fluctuation. For example, when SNR $=-10 \mathrm{~dB}$, SJR $=-30 \mathrm{~dB}, \epsilon=0.05$, and Laplacian noise is added as the jamming interference, the simulation results show that the variance under hypothesis $H_{0}$ is slightly larger than that under hypothesis $H_{1}$. However, the signal under hypothesis $H_{0}$ is modeled conservatively enough to ignore this effect. Therefore, for large $w, Z(w)$ can be modeled as a Gaussian random variable with

$$
\begin{aligned}
E\{Z(w)\} & =0 \\
\operatorname{var}\{Z(w)\} & =w \sigma_{1}^{2}
\end{aligned}
$$

under both the hypotheses.

When the received PN signal is sampled at a rate higher than the chip rate, we consider the use of a double median filtering scheme, in which the samples are first processed by a block median filter and then by a running median filter at a chip rate. The use of a running median filter can significantly reduce the correlation effect between the oversampled signals. For example, when SNR $=-5 \mathrm{~dB}, \mathrm{SJR}=-25 \mathrm{~dB}$, and $\epsilon=0.05$, the variance of the noise power is reduced from 19.0 to 1.84 with the use of double median filtering, while it is reduced only to 12.9 with the use of block median filtering of samples taken at three times the chip rate.

The test statistic of the double median filtering scheme is given by

$$
T(w)=\sum_{i=M_{R}-1}^{w+M_{R}-2} g_{R}\left(g_{B}\left(x_{i}\right)\right)
$$

Since

$$
s_{i M_{B}}=s_{i M_{B}+1}=\cdots=s_{i M_{B}+\left(M_{B}-1\right)}=c_{i+k} c_{i+l}
$$

we can rewrite $T(w)$ as

$$
\begin{aligned}
T(w) & =\sum_{i=M_{R}-1}^{w+M_{R}-2} g_{R}\left(c_{i+k} c_{i+l}+g_{B}\left(z_{i}\right)\right) \\
& =\sum_{i=M_{R}-1}^{w+M_{R}-2} g_{R}\left(s_{i}+\tilde{z}_{i}\right)
\end{aligned}
$$

where $\tilde{z}_{i}=g_{B}\left(z_{i}\right)$. This equation has the same form as (18) except that $z_{i}$ is replaced with $\tilde{z}_{i}$. Thus, $T(w)$ can be expressed as

$$
\begin{aligned}
T(w) & =\sum_{i=0}^{w-1}\left(s_{i+M_{R}-1}^{\prime}+\tilde{z}_{i+M_{R}-1}^{\prime}\right) \\
& =\sum_{i=0}^{w-1} s_{i+M_{R}-1}^{\prime}+\sum_{i=0}^{w-1} \tilde{z}_{i+M_{R}-1}^{\prime}
\end{aligned}
$$

where

$$
\begin{aligned}
& s_{i}^{\prime} \in\left\{s_{i}, s_{i+1}, \ldots, s_{i+M_{R}-1}\right\} \\
& \tilde{z}_{i}^{\prime} \in\left\{\tilde{z}_{i}, \tilde{z}_{i+1}, \ldots, \tilde{z}_{i+M_{R}-1}\right\} .
\end{aligned}
$$

Since the signal term $\sum_{i=0}^{w-1} s_{i+M_{R}-1}^{\prime}$ is equal to $S(w)$, the model [(31)] can also be applied to this case. Although the noise component $\tilde{z}_{i}^{\prime}$ s are in fact not i.i.d., they can be approximated as i.i.d. random variables with some variance $\sigma_{2}^{2}$ just as in the case that the samples are taken at a chip rate. For a sufficiently large value of $w, \sum_{i=0}^{w-1} \tilde{z}_{i+M_{R}-1}^{\prime}$ can also be approximated as a Gaussian random variable. Therefore, $\tilde{z}_{i}^{\prime}$ can also be assumed Gaussian.

\section{DeSIGN OF THE ACQUisition System}

By applying the models for the signal and noise component of the median filter output, the acquisition problem can be formulated as testing two simple hypotheses

$$
\begin{aligned}
& H_{0}: Y_{i} \sim \begin{cases}f\left(y-\hat{s}_{a}\right), & i \leq W_{b} \\
f\left(y-\hat{s}_{b}\right), & i>W_{b}\end{cases} \\
& H_{1}: Y_{i} \sim f\left(y-\hat{s}_{1}\right)
\end{aligned}
$$

where $f(y)$ is the pdf of the noise and jamming signals, approximated by

$$
f(y)=\frac{1}{\sqrt{2 \pi \sigma_{u}^{2}}} e^{-\frac{y^{2}}{2 \sigma_{u}^{2}}} .
$$


Here, $u$ is equal to one or two, respectively, if the samples are taken at a chip rate or at three times the chip rate.

The log-likelihood ratio test becomes

$$
\begin{aligned}
v_{i} & =\ln \frac{f\left(y_{i}-\hat{s}_{1}\right)}{f\left(y_{i}-\hat{s}_{0 i}\right)} \\
& = \begin{cases}\frac{\hat{s}_{1}-\hat{s}_{a}}{\sigma_{u}^{2}}\left(y_{i}-\frac{\hat{s}_{a}+\hat{s}_{1}}{2}\right), & i \leq W_{b} \\
\frac{\hat{s}_{1}-\hat{s}_{b}}{\sigma_{u}^{2}}\left(y_{i}-\frac{\hat{s}_{b}+\hat{s}_{1}}{2}\right), & i>W_{b} .\end{cases}
\end{aligned}
$$

Since the samples are assumed to be i.i.d., the decision statistic $\lambda(w)$ becomes

$$
\lambda(w)=\sum_{i=0}^{w-1} v_{i+M_{R}-1} .
$$

With the decision statistic, we can design the fixed sample size test by

$$
\lambda\left(W_{F}\right) \begin{cases}\geq t_{F} & \Rightarrow \text { Accept } H_{1} \\ <t_{F} & \Rightarrow \text { Accept } H_{0} .\end{cases}
$$

Given false-alarm probability $\alpha$ and true detection probability $\beta$, the required sample size $W_{F}$ and the threshold $t_{F}$ can be calculated [26]. For ease of description, let us define

$$
\begin{aligned}
\mu_{a} & =\left(\hat{s}_{1}-\hat{s}_{a}\right)^{2} /\left(2 \sigma_{u}^{2}\right) \\
\mu_{b} & =\left(\hat{s}_{1}-\hat{s}_{b}\right)^{2} /\left(2 \sigma_{u}^{2}\right) \\
\sigma_{a}^{2} & =\left(\hat{s}_{1}-\hat{s}_{a}\right)^{2} / \sigma_{u}^{2} \\
\sigma_{b}^{2} & =\left(\hat{s}_{1}-\hat{s}_{b}\right)^{2} / \sigma_{u}^{2} .
\end{aligned}
$$

When $W_{F} \leq W_{b}, W_{F}$ and $t_{F}$ are determined by

$$
\begin{aligned}
W_{F} & =\frac{\sigma_{a}^{2}}{2 \mu_{a}}\left[\Phi^{-1}(\alpha)+\Phi^{-1}(1-\beta)\right]^{2} \\
t_{F} & =\frac{\sqrt{W_{F}} \sigma_{a}}{2}\left[\Phi^{-1}(1-\beta)-\Phi^{-1}(\alpha)\right]
\end{aligned}
$$

where $\Phi^{-1}(\cdot)$ is the inverse function of the Gaussian distribution function. When $W_{F}>W_{b}$, we have

$$
\begin{aligned}
W_{F}= & \frac{1}{8 \mu_{b}^{2}}\left[-8 \mu_{b} \mu_{w}+\sigma_{b}^{2} \rho^{2}\right. \\
& \left.+\sqrt{\left(8 \mu_{b} \mu_{w}-\sigma_{b}^{2} \rho^{2}\right)^{2}-16 \mu_{b}^{2}\left(4 \mu_{w}^{2}-\rho^{2} \sigma_{w}^{2}\right)}\right] \\
t_{F}= & \frac{1}{2} \sqrt{W_{F} \sigma_{b}^{2}+\sigma_{w}^{2}} \cdot\left[\Phi^{-1}(1-\beta)-\Phi^{-1}(\alpha)\right]
\end{aligned}
$$

where $\mu_{w}=\left(\mu_{a}-\mu_{b}\right) W_{b}, \sigma_{w}^{2}=\left(\sigma_{a}^{2}-\sigma_{b}^{2}\right) W_{b}$, and $\rho^{2}=$ $\left[\Phi^{-1}(\alpha)+\Phi^{-1}(1-\beta)\right]^{2}$.

When the input signal is oversampled, it is not easy to analytically calculate the value of $\sigma_{2}^{2}$. However, when $\alpha=1-\beta$, the threshold for the test becomes zero by (48) and (49) without the need of $\sigma_{2}^{2}$. Thus, the required sample size $W_{F}$ can easily be obtained for performance comparison.

\section{Performance Evaluation}

To verify the validity of the approximated model for the output of the median filter, analytical results are compared with experimental ones. Figs. 7 and 8 plot the detection probability in terms of the sample size $W_{F}$ when 1) $\epsilon=0.03, \mathrm{SNR}=-5 \mathrm{~dB}$, $\mathrm{SJR}=-25 \mathrm{~dB}$, and $m=9$ and 2) $\epsilon=0.03, \mathrm{SNR}=-10 \mathrm{~dB}$, $\mathrm{SJR}=-30 \mathrm{~dB}$, and $m=10$, respectively. Note that the samples are taken at a chip rate and processed by a running median

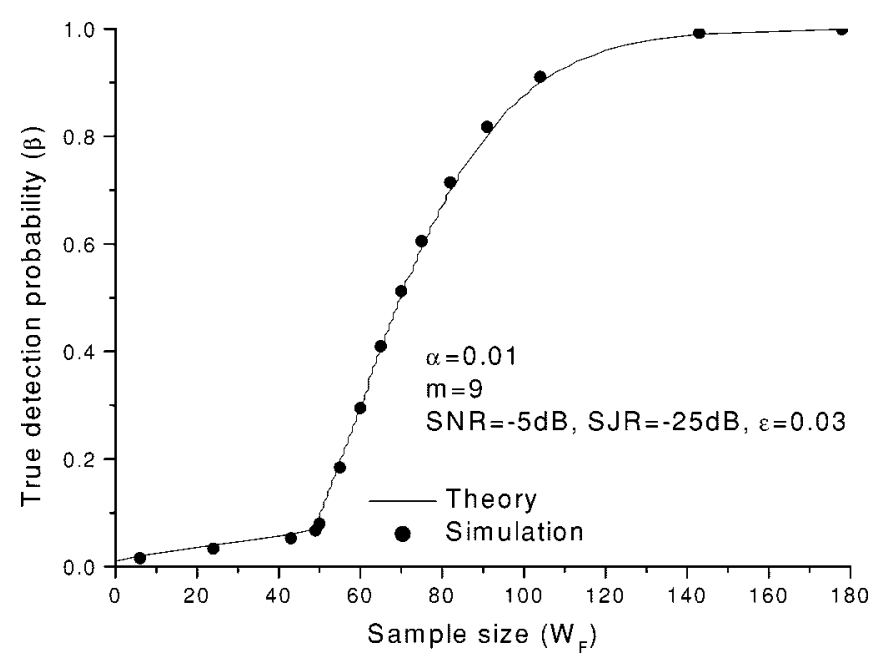

(a)

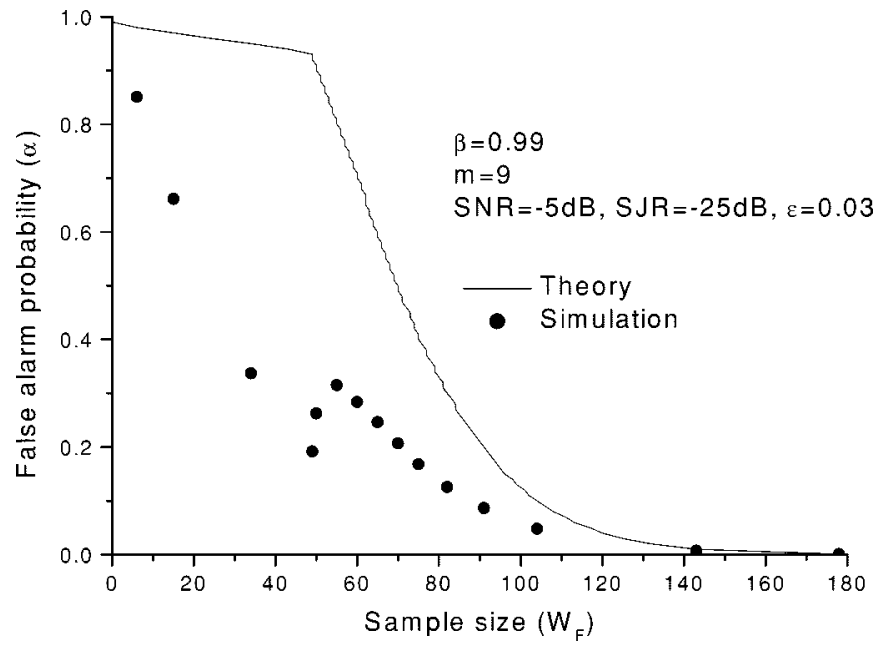

(b)

Fig. 7. Detection probability of the proposed system when $m=9$. (a) $\alpha=$ 0.01. (b) $\beta=0.99$.

filter. The solid lines depict the analytical results and the dots the simulation results. In Figs. 7(a) and 8(a), the true detection probability $\beta$ is depicted when the false-alarm probability $\alpha$ is set to a fixed value of 0.01 and 0.001 , respectively. Using the required sample size $W_{F}$ from (48) and (49) for a designed value of $\beta$, the simulation results are compared with the analytic design. It can be seen that the simulation results agree very well with the analytic design. Figs. 7(b) and 8(b) plot the false-alarm probability $\alpha$ when the true detection probability $\beta$ is fixed to 0.99 and 0.999 , respectively. It can be seen that the actual false-detection probability is lower than the designed one, due to the use of the conservative signal model $\bar{S}(w)$. The discrepancy between the actual false-detection probability and the designed one decreases as the SNR decreases. However, the analytic design always guarantees the designed performance. The temporary increase in the false-alarm probability after $w=W_{b}$ in Fig. 7(b) is also due to the use of the conservative signal model $\hat{s}_{a}=0.75$, which makes the SNR of the decision statistic

$$
\operatorname{SNR}_{\lambda}(w)=\frac{E\{\lambda(w)\}^{2}}{\operatorname{var}\{\lambda(w)\}}
$$




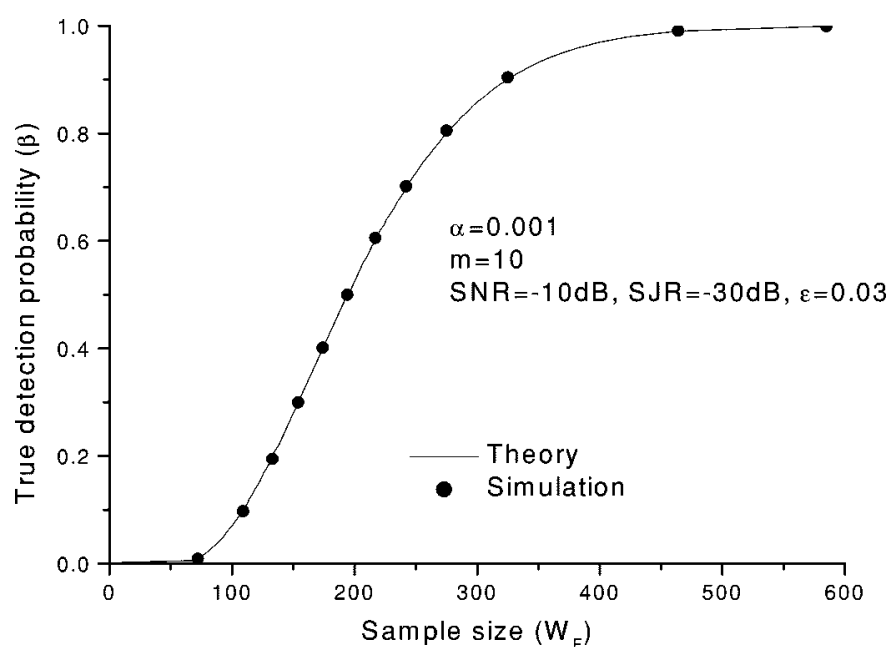

(a)

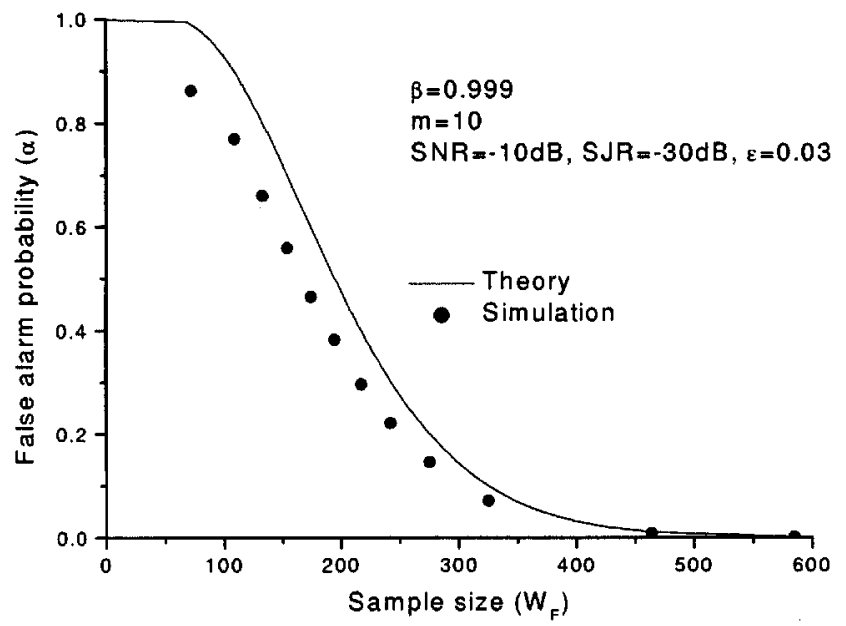

(b)

Fig. 8. Detection probability of the proposed system when $m=10$.

which temporarily decreases right after the signal model changes from $\hat{s}_{a}=0.75$ to $\hat{s}_{b}=0.0$ at $w=W_{b}$.

The performance of an acquisition scheme is generally evaluated in terms of the mean acquisition time, probability of successful acquisition within a given time, and/or implementation complexity. Since the mean acquisition time of a sliding correlator-type acquisition scheme increases linearly in proportion to the required sample size by the fixed sample size test, it can be used for the purpose of performance evaluation (refer to (8) in [26]). In general, the required sample sizes for hypotheses $H_{0}$ and $H_{1}$ are different from each other, except when $\alpha=1-\beta$. For ease of comparison, we consider the case when $\alpha=1-\beta$ in the following discussion.

Fig. 9 depicts the required sample size $W_{F}$ when samples are taken at a chip rate and a running median filter with $M_{R}=3$ is employed. The required sample size to satisfy $\alpha=0.01$ and $\beta=0.99$ is depicted as a function of the jamming duty factor $\epsilon$, when SNR $=-5 \mathrm{~dB}$, and SJR $=-15 \mathrm{~dB}$ and $-25 \mathrm{~dB}$ using the $m$-sequence A in Table I. Since the fixed sample size test uses the same number of samples for both hypotheses, Fig. 9 plots the required sample size for hypothesis $H_{1}$. It can be seen that the analytic design can guarantee the desired performance and agrees well with the simulation result.

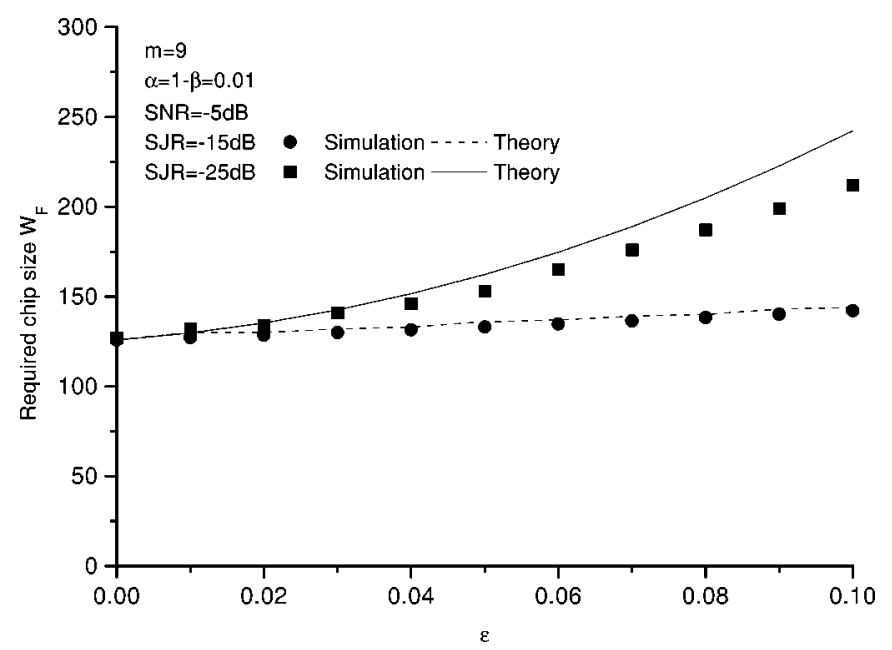

Fig. 9. Required sample sizes when $\alpha=1-\beta=0.01$.

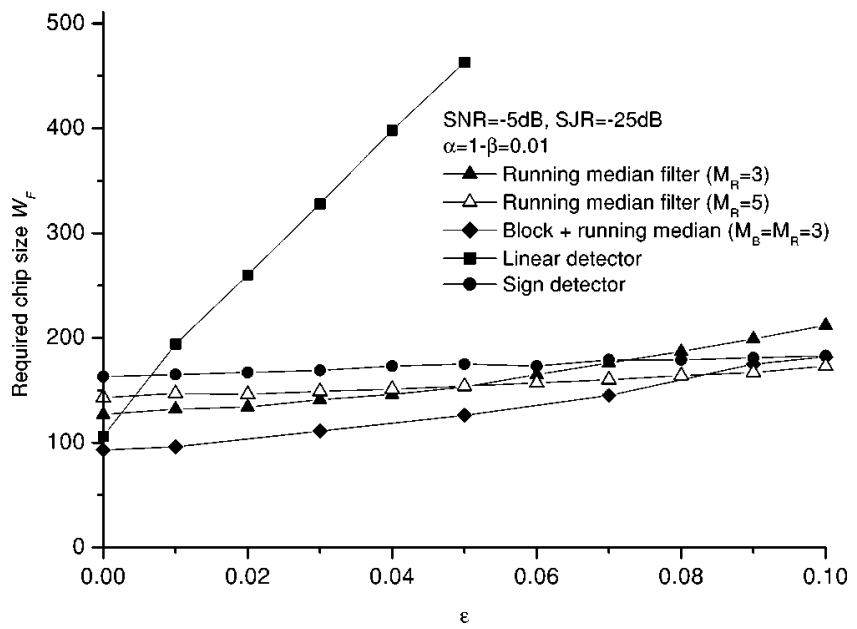

(a)

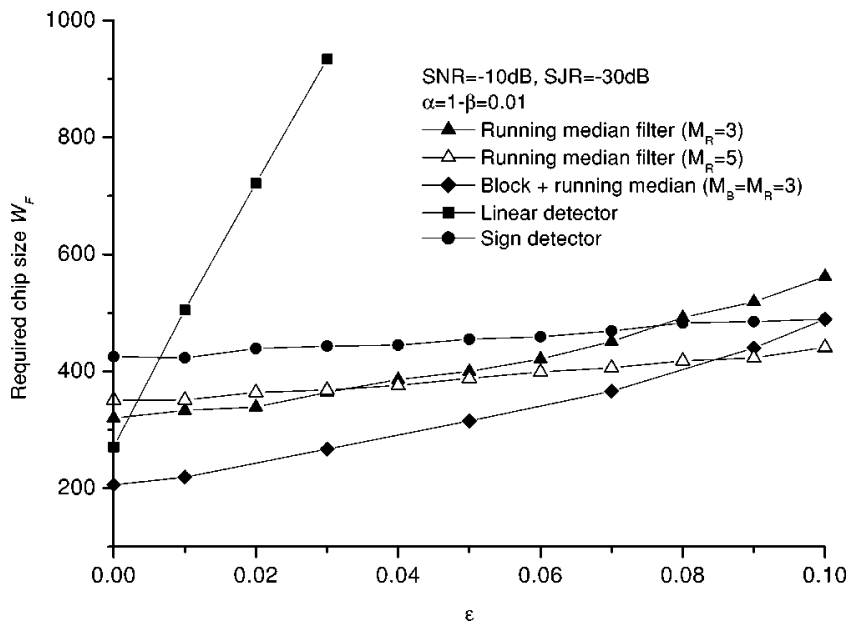

(b)

Fig. 10. Performance comparison with the conventional schemes. (a) $m=9$. (b) $m=10$.

The performance of the proposed acquisition schemes is compared with that of the conventional linear correlator-based acquisition scheme and the sign detector-based scheme. Fig. 10 depicts the required sample sizes when $m=9, \mathrm{SNR}=-5 \mathrm{~dB}$ and $\mathrm{SJR}=-25 \mathrm{~dB}$, and when $m=10, \mathrm{SNR}=-10 \mathrm{~dB}$, and 
$\mathrm{SJR}=-30 \mathrm{~dB}$. It can be seen that the performance of the linear correlator-based acquisition scheme degrades rapidly as $\epsilon$ increases. The sign detector-based acquisition scheme can provide performance robust to variation of $\epsilon$, but it requires more samples than the proposed ones when $\epsilon$ is not too large. The proposed scheme with double median filtering outperforms all the other tested detectors when $\epsilon<0.08$. Note that it performs better than the linear correlator-based scheme even when $\epsilon=0$. This is because more information is available to the detector by taking three samples per chip.

In practice, it is not easy to make a wide-band jamming signal with high duty cycle. Thus, the use of the median filter with a small $M_{R}$ may be sufficient for most of the cases. However, when $\epsilon$ becomes large, the performance degradation can be overcome by increasing the window size $M_{R}$. As can be seen in Fig. 10, the proposed scheme with $M_{R}=5$ can provide performance more robust to $\epsilon$ than the one with $M_{R}=3$. Thus, it outperforms the sign detector-based scheme even when $\epsilon=0.1$.

\section{CONCLUSION}

In this paper, we have proposed PN sequence acquisition schemes robust to wide-band jamming interference. To reduce the effect of the wide-band impulse noise, we considered the use of a simple median filter as a robust preprocessor. Depending on the oversampling rate, two types of median filtering were considered. Since the use of nonlinear memory operation in the median filtering process makes analytic design of the detector complicated, a simple model has been proposed for the output of the median filters. The analytic design has been verified by computer simulation. Numerical results showed that the proposed schemes provide acquisition performance robust to wide-band jamming interference without significant increase of the implementation complexity.

\section{REFERENCES}

[1] R. Pickholtz, D. Schilling, and L. Milstein, "Theory of spread-spectrum communications-A tutorial," IEEE Trans. Commun., vol. COM-30, pp. 855-884, May 1982.

[2] M. Simon, J. Omura, R. Scholtz, and B. Levitt, Spread Spectrum Communications Handbook, rev. ed. New York: McGraw-Hill, 1994.

[3] B. Aazhang and H. V. Poor, "Performance of DS/SSMA communications in impulsive channels-Part I: Linear correlation receivers," IEEE Trans. Commun., vol. COM-35, pp. 1179-1187, Nov. 1987.

[4] X. Gui and T. S. Ng, "Performance of DS SS system under on-off wideband jamming," Electron. Lett., vol. 33, pp. 557-559, Mar. 1997.

[5] L. B. Milstein, "Interference rejection in spread spectrum communications," Proc. IEEE, vol. 76, no. 6, pp. 657-671, 1988.

[6] J. G. Proakis, "Interference suppression in spread spectrum systems," in Proc. ISSSTA, 1996, pp. 259-266.

[7] B. Aazhang and H. V. Poor, "Performance of DS/SSMA communications in impulsive channels-Part II: Hard-limiting correlator receivers," IEEE Trans. Commun., vol. 36, pp. 88-97, Jan. 1988.

[8] G. Stüber, "Soft-limiter receiver for coded DS/DPSK systems," IEEE Trans. Commun., vol. 38, Jan. 1990.

[9] J. Chao and C. Lee, "An efficient direct-sequence signal detector based on Depster-Shafer theory," IEEE Trans. Commun., vol. 38, pp. 868-874, June 1990.

[10] A. Polydoros and C. Weber, "A unified approach to serial search spreadspectrum code acquisition-Part I \& II," IEEE Trans. Commun., vol. COM-32, pp. 542-560, May 1984.

[11] J. Holmes and C. Chen, "Acquisition time performance of PN spreadspectrum systems," IEEE Trans. Commun., vol. COM-25, pp. 778-783, Aug. 1977.
[12] S. David and E. Dodds, "Acquisition time distribution for spread-spectrum receivers," IEEE J. Select. Areas Commun., vol. 8, pp. 800-807, June 1990.

[13] E. Siess and C. Weber, "Acquisition of direct sequence signals with modulation and jamming," IEEE J. Select. Areas Commun., vol. SAC-4, pp. 254-272, Mar. 1986.

[14] H. V. Poor, An Introduction to Signal Detection and Estimation. Berlin, Germany: Springer-Verlag, 1994.

[15] A. Bovik, T. Huang, and D. Munson, "A generalization of median filtering using linear combinations of order statistics," IEEE Trans. Acoust., Speech, Signal Processing, vol. ASSP-31, pp. 1342-1350, Dec. 1983.

[16] J. Saniie, K. Donohue, and N. Bilgutay, "Order statistic filters as postdetection processors," IEEE Trans. Acoust., Speech, Signal Processing, vol. 38, pp. 1722-1731, Oct. 1990.

[17] A. Swami and B. Sadler, "TDE, DOA and related parameter estimation problems in impulsive noise," in IEEE Workshop Higher Order Statistics, 1997, pp. 273-277.

[18] Y.-H. Lee and S.-J. Kim, "Robust signal detection using order statistic prefilters," IEICE Trans. Commun., vol. E81-B, pp. 520-524, Mar. 1998.

[19] J. Iinatti, "Performance comparison of DS signal matched filter acquisition using median filter and transversal filter as an aiding device in pulsed tone jamming," in Proc. MILCOM'95, 1995, pp. 1190-1194.

[20] K. K. Chawla and D. V. Sarwate, "Acquisition of pn sequences in chip synchronous ds/ss systems using a random sequence model and the sprt," IEEE Trans. Commun., vol. 42, no. 6, pp. 2325-2334, 1993.

[21] P. Huber, "A robust version of the probability ratio test," Ann. Math. Statist., vol. 36, pp. 1753-1758, Dec. 1965.

[22] D. Torrieri, "The performance of five different metrics against pulsed jamming," IEEE Trans. Commun., vol. COM-34, pp. 200-204, Feb. 1986.

[23] J. Astola and T. Campbell, "On computation of the running median," IEEE Trans. Acoust., Speech, Signal Processing, vol. 37, pp. 572-574, Apr. 1989.

[24] Y.-H. Lee and S. Tantaratana, "Robust detection of a constant signal using order statistic filters," in Proc. Conf. Information Science and Systems, Mar. 1989, pp. 28-33.

[25] H. David, Order Statistics, 2nd ed. New York: Wiley, 1981.

[26] Y.-H. Lee and S. Tantaratana, "Sequential acquisition of PN sequences for DS/SS communications: Design and performance," IEEE J. Select. Areas Commun., vol. 10, pp. 750-759, May 1992.

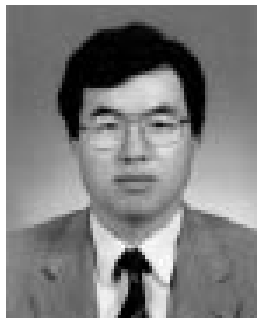

Yong-Hwan Lee (S'77-M'89) received the B.S. degree from Seoul National University, Korea, in 1977, the M.S. degree from the Korea Advanced Institute of Science and Technology (KAIST), Korea, in 1980, and the Ph.D. degree from the University of Massachusetts, Amherst, in 1989, all in electrical engineering.

From 1980 to 1985, he was with the Korea Agency for Defense Development, where he was involved in development of shipboard weapon fire control systems. From 1989 to 1994, he worked for Motorola as a Principal Engineer, where he engaged in research and development of data transmission systems including high-speed modems. In 1994, he joined the School of Electrical Engineering, Seoul National University, as a Faculty Member. His research areas are wired/wireless transmission systems including spread-spectrum systems, robust signal detection/estimation theory, and signal processing for communications.

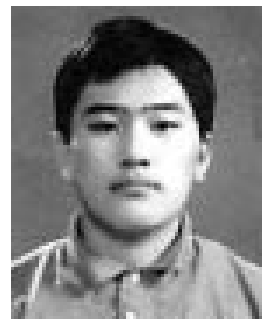

Seung-Jun Kim (S'97) received the B.S. and M.S. degrees in electrical engineering from Seoul National University, Seoul, Korea, in 1996 and 1998, respectively. $\mathrm{He}$ is now pursuing the $\mathrm{Ph} . \mathrm{D}$. degree in electrical engineering at the University of California, Santa Barbara.

His current research interests are in detection theory and spread-spectrum communications. 